\title{
Progressive Taxation and Macroeconomic (In)stability with Utility-Generating Government Spending*
}

\author{
Shu-Hua Chen ${ }^{\dagger}$ \\ National Taipei University
}

\author{
Jang-Ting Guo \\ University of California, Riverside
}

April 22, 2013

\begin{abstract}
We examine the theoretical interrelations between progressive income taxation and macroeconomic (in)stability in an otherwise standard one-sector real business cycle model with utility-generating government purchases of goods and services. When private and public consumption expenditures are complements in the household utility and the tax schedule is progressive, we analytically show that the economy exhibits indeterminacy and sunspots if and only if the degree of government-spending preference externality is higher than a critical threshold. Unlike traditional Keynesian-type stabilization policies, raising the tax progressivity may destabilize this version of our model by generating endogenous cyclical fluctuations. Moreover, the economy always displays saddle-path stability and equilibrium uniqueness under utility substitutability between private and public consumptions and progressive taxation.
\end{abstract}

Keywords: Progressive Income Taxation, Equilibrium (In)determinacy, Utility-Generating Government Spending, Business Cycles.

JEL Classification: E32, E62.

\footnotetext{
${ }^{*}$ We thank Been-Lon Chen, Hung-Ju Chen, Sharon Harrison, Chia-Hui Lu, Victor Ortego-Marti, Richard Suen, Yi-Chan Tsai, and seminar participants at Chinese Economic Association in North America at the ASSA Meetings and National Taiwan University for helpful comments and suggestions. Part of this research was conducted while Guo was a visiting research fellow of economics at Academia Sinica, Taipei, Taiwan, whose hospitality is greatly appreciated. Of course, all remaining errors are our own.

${ }^{\dagger}$ Department of Economics, National Taipei University, 151 University Rd., San Shia District, New Taipei City, 23741 Taiwan, 886-2-8674-1111 ext. 67165, Fax: 886-2-2673-9880, E-mail: shchen@mail.ntpu.edu.tw.

${ }^{\ddagger}$ Corresponding Author. Department of Economics, 3133 Sproul Hall, University of California, Riverside, CA, 92521 USA, 1-951-827-1588, Fax: 1-951-827-5685, E-mail: guojt@ucr.edu.
} 


\section{Introduction}

The relationship between government purchases of goods and services versus agents' private consumption is an important aspect in understanding the aggregate effects of a fiscal policy rule within dynamic general equilibrium macroeconomic models. Specifically, private and public consumption expenditures may enter the household's utility function as Edgeworth complements or substitutes. With the noted exceptions of Cazzavillan (1996), Zhang (2000), Baier and Glomm (2001), Fernández, Novales and Ruiz (2004), Chen (2006), and Guo and Harrison (2008), previous theoretical research has studied representative-agent models under productive flow of government spending ${ }^{1}$ or stock of public capital. ${ }^{2}$ Motivated by this gap in the existing literature, we consider a prototypical one-sector real business cycle (RBC) model with two prevalent features observed in developed economies: progressive income taxation ${ }^{3}$ and utility-generating government purchases, and analytically explore the interrelations between tax progressivity and equilibrium (in)determinacy. ${ }^{4}$ To our knowledge, this is the first piece of work that explores such an interesting research topic not only for its theoretical relevance, but also for its broad implications for the design, evaluation and implementation of tax policies.

In this paper, we systematically examine the stabilization effects of Guo and Lansing's (1998) progressive tax formulation in an otherwise standard one-sector RBC model with balanced budget and utility-generating public expenditures. Per the empirical findings of Ni (1995), our analysis examines a constant-relative-risk-aversion (CRRA) Cobb-Douglas utility specification that postulates government spending as a positive preference externality. As it turns out, the (local) stability properties of our model's unique interior steady state depend crucially on (i) the utility complementarity or substitutability between private and public consumptions, (ii) the slope parameter of the tax schedule that governs its progressivity attribute, and (iii) the degree of government-purchases preference externality.

When government spending is complementary to private consumption in the household utility and the tax policy is progressive, we derive the necessary and sufficient condition under which our model exhibits an indeterminate steady state and endogenous cyclical fluctuations driven by animal spirits or sunspots. In particular, the degree of preference externality from

\footnotetext{
${ }^{1}$ See, for example, Barro (1990), Barro and Sala-i-Martin (1992), Cazzavillan (1996), Glomm and Ravikumar (1997), Turnovsky (1999), Zhang (2000), Palivos, Yip and Zhang (2003), Slobodyan (2006), Hu, Ohdoi and Shimomura (2008), and Chen and Guo (2013a, 2013b) among others.

${ }^{2}$ See, for example, Futagami, Morita and Shibata (1993), Glomm and Ravikumar (1994), Turnovsky (1997), Baier and Glomm (2001), Chen (2006), Greiner (2006, 2007), and Agénor (2011), among others.

${ }^{3}$ By contrast, Cazzavillan (1996), Zhang (2000), Baier and Glomm (2001), Fernández, Novales and Ruiz (2004), Chen (2006), and Guo and Harrison (2008) all postulate a constant tax rate of income.

${ }^{4}$ See Benhabib and Farmer (1999) for an excellent survey of the RBC-based indeterminacy literature.
} 
public expenditures needs to be higher than a critical value that can be analytically expressed as a function of other structural parameters. Start the economy from its steady state, and consider a slight deviation caused by agents' optimistic anticipation about an expansion in future economic activities. Acting upon this belief, the representative household will reduce consumption and raise investment today. This in turn leads to another dynamic trajectory with higher future output, private consumption, and income tax rate because of progressive taxation. Through the government's balanced-budget constraint, the level of public spending also rises, which will then produce a further increase in future private consumption since private and public consumption expenditures are Edgeworth complements. We show that the aftertax return on investment is monotonically increasing along this alternative transitional path if and only if the government-spending preference externality exceeds the requisite threshold. As a result, agents' initial rosy expectations on the economy's future are validated as a selffulfilling equilibrium. Moreover, in sharp contrast to existing studies with useless or wasteful government purchases of goods and services ${ }^{5}$, raising the tax progressivity ceteris paribus may transform our model's steady state from a saddle point into a sink provided the degree of utility complementarity between private and public consumptions is sufficiently strong. It follows that unlike traditional Keynesian-type stabilization policies, a more progressive tax schedule may destabilize the economy by generating belief-driven business cycle fluctuations.

When government spending is substitutable with private consumption in the household utility and the tax policy is progressive, we find that the mechanism described in the proceeding formulation that makes for multiple equilibria, i.e. an increase of the equilibrium after-tax marginal product of capital in response to higher expenditures of today's investment, will not be realized in that higher public expenditures now lower the marginal utility of private consumption. It follows that our model economy always exhibits saddle-path stability and equilibrium uniqueness in this setting. Finally, the same stability/uniqueness result continues to hold when there is no government-purchases preference externality, regardless of the level of tax progressivity under consideration; or when the income tax rate is a fixed constant, no matter whether private and public consumptions are Edgeworth complements or substitutes.

The remainder of this paper is organized as follows. Section 2 describes the model and analyzes its equilibrium conditions. Section 3 examines the theoretical interrelations between tax progressivity, government-spending preference externality and our model's local stability properties. Section 4 concludes.

\footnotetext{
${ }^{5}$ See, for example, Schmitt-Grohé and Uribe, (1997), Guo and Lansing (1998), and Christiano and Harrison (1999), among others.
} 


\section{The Economy}

We incorporate utility-generating government purchases of goods and services into an otherwise standard one-sector real business cycle (RBC) model under the progressive income tax policy á la Guo and Lansing (1998). Households live forever, and derive utilities from private consumption, public expenditures and leisure. Based on the empirical findings of Ni (1995), our analysis examines a constant-relative-risk-aversion (CRRA) Cobb-Douglas utility specification that postulates government spending as a positive preference externality. On the production side, each competitive firm produces output using a constant returns-to-scale technology with capital and labor as inputs. We further assume that there are no fundamental uncertainties present in the economy.

\subsection{Firms}

There is a continuum of identical competitive firms, with the total number normalized to one. The representative firm produces output $Y_{t}$, using physical capital $K_{t}$ and labor hours $H_{t}$ as inputs, with a constant returns-to-scale Cobb-Douglas production function

$$
Y_{t}=K_{t}^{\alpha} H_{t}^{1-\alpha}, \quad 0<\alpha<1
$$

Under the assumption that factor markets are perfectly competitive, the firm's profit maximization conditions are given by

$$
\begin{gathered}
r_{t}=\alpha \frac{Y_{t}}{K_{t}}, \\
w_{t}=(1-\alpha) \frac{Y_{t}}{H_{t}},
\end{gathered}
$$

where $r_{t}$ is the capital rental rate and $w_{t}$ is the real wage. In addition, $\alpha$ and $1-\alpha$ represent the capital and labor share of national income, respectively.

\subsection{Households}

The economy is populated by a unit measure of identical infinitely-lived households. Each household is endowed with one unit of time and maximizes a discounted stream of utilities over its lifetime 


$$
\int_{0}^{\infty}\left[\frac{\left(C_{t}^{\theta_{1}} G_{t}^{\theta_{2}}\right)^{1-\sigma}}{1-\sigma}-A \frac{H_{t}^{1+\gamma}}{1+\gamma}\right] e^{-\rho t} d t, \quad A, \sigma>0, \quad \sigma \neq 1, \quad \text { and } \quad \gamma \geq 0
$$

where $\rho>0$ is the subjective discount rate, $C_{t}$ is private consumption, $H_{t}$ is hours worked, and $\gamma$ governs the inverse of the labor supply elasticity. ${ }^{6}$ Moreover, $G_{t}$ denotes the flow of government spending on goods and services that are determined outside the individual household's control. Per the empirical results of Ni (1995), the period utility function in our model (i) is increasing and strictly concave with respect to private consumption, thus $\theta_{1}>0$ and $\theta_{1}(1-\sigma)<1$; (ii) is increasing in public consumption, thus $\theta_{2}>0$ indicating the presence of a positive preference externality; and (iii) exhibits linear homogeneity in "effective consumption" $C_{t}^{\theta_{1}} G_{t}^{\theta_{2}}$, thus $\theta_{1}+\theta_{2}=1$ (see also Bean [1986], and Campbell and Mankiw [1990]). ${ }^{7}$ Finally, when $\sigma<(>) 1$, the marginal utility of private consumption increases (decreases) with respect to government spending, hence $C_{t}$ and $G_{t}$ are Edgeworth complements (substitutes).

The budget constraint faced by the representative household is given by

$$
\dot{K}_{t}=\left(1-\tau_{t}\right)\left(r_{t} K_{t}+w_{t} H_{t}\right)-\delta K_{t}-C_{t}, \quad K_{0}>0 \text { given, }
$$

where $K_{t}$ is the household's capital stock and $\delta \in(0,1)$ is the capital depreciation rate. Households derive income by providing capital and labor services to firms, taking factor prices $r_{t}$ and $w_{t}$ as given. As in Guo and Lansing (1998), we postulate that the income tax rate $\tau_{t}$ takes the form

$$
\tau_{t}=1-\eta\left(\frac{Y^{*}}{Y_{t}}\right)^{\phi}, \eta \in(0,1) \text { and } \phi \in[0,1)
$$

where $Y_{t}$ represents the household's taxable income $\left(=r_{t} K_{t}+w_{t} L_{t}\right)$, and $Y^{*}$ denotes the steady-state level of per capita income, which is taken as given by each agent. The parameters $\eta$ and $\phi$ govern the level and slope of the tax schedule, respectively. When $\phi>0$, the tax rate

\footnotetext{
${ }^{6}$ The specification with $\gamma=0$ draws on the formulation of indivisible labor as in Hansen (1985) and Rogerson (1988).

${ }^{7} \mathrm{Ni}$ (1995) considers the linear and Cobb-Douglas specifications of effective consumption, which specifies how private and public consumptions are combined into a composite good that enters the CRRA-variety utility function $U\left(C_{t}, G_{t}\right)$. When effective consumption is postulated as a linear function, the Edgeworth complementarity between $C_{t}$ and $G_{t}$ implies that $U(\cdot)$ is decreasing in government expenditures. This violates a standard assumption on the household preferences, and generates more unstable point estimates compared to those under the Cobb-Douglas form of effective consumption. Moreover, estimation results based on the generalized CES formulation of effective consumption show that the Cobb-Douglas specification is more appropriate than the linear alternative. Based on these findings, we adopt the CRRA Cobb-Douglas preference formulation (equation 4) in our analysis.
} 
$\tau_{t}$ rises with the household's taxable income $Y_{t}$. When $\phi=0$, all households face the constant tax rate $1-\eta$ regardless of their taxable income.

With regard to the progressivity features of the above taxation scheme, we first note that the marginal tax rate $\tau_{m t}$, defined as the change in taxes paid by the household divided by the change in its taxable income, is given by

$$
\tau_{m t} \equiv \frac{\partial\left(\tau_{t} Y_{t}\right)}{\partial Y_{t}}=1-\eta(1-\phi)\left(\frac{Y^{*}}{Y_{t}}\right)^{\phi} .
$$

In addition, our analysis is restricted to environments with $0<\tau_{t}, \tau_{m t}<1$ such that (i) the government does not have access to lump-sum taxes or transfers, (ii) the government cannot confiscate all productive resources, and (iii) households have incentive to supply factor services to the firm's production process. In the model's steady state, the preceding considerations imply that $0<\eta<1$ and that $\frac{\eta-1}{\eta}<\phi<1$, where $\frac{\eta-1}{\eta}<0$.

Next, in order to satisfy the second-order conditions of the representative agent's dynamic optimization problem, its budget constraint (5) needs to be jointly concave in the state and control variables, i.e. $K_{t}, C_{t}$ and $H_{t}$. We find that this requirement, together with $\eta \in(0,1)$ and $\phi<1$, yields a more binding lower bound on the tax-slope parameter $\phi \geq 0$. Given these restrictions on $\eta$ and $\phi$, it is straightforward to show that when $\phi>0$, the marginal tax rate is higher than the average tax rate given by (6). In this case, the tax schedule is said to be "progressive". When $\phi=0$, the average and marginal tax rates coincide at the level $1-\eta$, and the tax schedule is said to be "flat".

We postulate that agents take into account the way in which the tax schedule affects their earnings when they decide how much to consume, invest and work over their lifetimes. Therefore, it is the marginal tax rate of income $\tau_{m t}$ that governs the household's economic decisions. The first-order conditions for the representative agent with respect to the indicated variables and the associated transversality condition (TVC) are 


$$
\begin{aligned}
& C_{t}: \theta_{1} C_{t}^{\theta_{1}(1-\sigma)-1} G_{t}^{\theta_{2}(1-\sigma)}=\lambda_{t}, \\
& H_{t} \quad: \quad \frac{A H_{t}^{\gamma}}{\lambda_{t}}=\underbrace{\eta(1-\phi)\left(\frac{Y^{*}}{Y_{t}}\right)^{\phi}}_{\left(1-\tau_{m t}\right)} w_{t}, \\
& K_{t}: \quad \quad-\frac{\dot{\lambda}_{t}}{\lambda_{t}}=\underbrace{\eta(1-\phi)\left(\frac{Y^{*}}{Y_{t}}\right)^{\phi}}_{\left(1-\tau_{m t}\right)} r_{t}-(\rho+\delta), \\
& T V C \quad: \quad \lim _{t \rightarrow \infty} e^{-\rho t} \lambda_{t} K_{t}=0,
\end{aligned}
$$

where $\lambda_{t}>0$ is the the Lagrange multiplier on the budget constraint (5), (9) equates the slope of the household's indifference curve to the after-tax real wage, (10) is the modified consumption Euler equation that takes into account the effect of public expenditures on the marginal utility of private consumption, and (11) is the transversality condition. Notice that under the restrictions on $\eta$ and $\phi$ specified above, equations (8)-(10) are not only necessary, but also sufficient conditions for the unique global maximum of the household's optimization problem.

\subsection{Government}

The government sets the tax rate $\tau_{t}$ according to (6), and balances its budget at each point in time. Hence, its instantaneous budget constraint is given by

$$
G_{t}=\tau_{t} Y_{t}
$$

where government spending on goods and services $G_{t}$ in turn contributes to the household's utilities. With the government, the aggregate resource constraint for the economy is

$$
C_{t}+\dot{K}_{t}+\delta K_{t}+G_{t}=Y_{t}
$$

\section{Macroeconomic (In)stability}

To facilitate the analysis of our model's local stability properties, we make the following logarithmic transformation of variables: $k_{t} \equiv \log \left(K_{t}\right)$ and $c_{t} \equiv \log \left(C_{t}\right)$. It is straightforward to show that our model exhibits a unique interior steady state given by 


$$
k^{*}=\frac{\log \left\{\frac{\left[\Delta(1-\eta)^{\theta_{2}(1-\sigma)}\left(x_{2} / x_{1}\right)^{\theta_{1}(1-\sigma)-1}\right]^{1-\alpha}}{x_{1}^{\alpha+\gamma+\sigma(1-\alpha)}}\right\}}{(1-\alpha)(\gamma+\sigma)}
$$

and

$$
c^{*}=\frac{\log \left\{\frac{\left[\Delta(1-\eta)^{\theta_{2}(1-\sigma)}\left(x_{2} / x_{1}\right)^{\gamma-\theta_{2}(1-\sigma)}\right]^{1-\alpha}}{x_{1}^{\alpha(1+\gamma)}}\right\}}{(1-\alpha)(\gamma+\sigma)},
$$

where

$$
\Delta \equiv \frac{\eta \theta_{1}(1-\alpha)(1-\phi)}{A}>0, x_{1} \equiv \frac{\rho+\delta}{\alpha \eta(1-\phi)}>0 \text { and } x_{2} \equiv \frac{\rho+[1-\alpha(1-\phi)] \delta}{\alpha(1-\phi)}>0 .{ }^{8}
$$

The remaining endogenous variables at the economy's steady state can then be derived accordingly.

Next, in the neighborhood of this steady state, our model's equilibrium conditions can be approximated by the following log-linearized dynamical system:

$$
\left[\begin{array}{c}
\dot{k}_{t} \\
\dot{c}_{t}
\end{array}\right]=\underbrace{\left[\begin{array}{ll}
\mathbf{J}_{11} & \mathbf{J}_{12} \\
\mathbf{J}_{21} & \mathbf{J}_{22}
\end{array}\right]}_{\mathbf{J}}\left[\begin{array}{c}
k_{t}-k^{*} \\
c_{t}-c^{*}
\end{array}\right], \quad k_{0} \text { given }
$$

where

$$
\begin{aligned}
& \mathbf{J}_{11}=\eta\left[\frac{\alpha(1+\gamma)(1-\phi)}{\Psi}-1\right] x_{1}+x_{2}, \\
& \mathbf{J}_{12}=\frac{\eta(\alpha-1)(1-\phi)\left[1-\theta_{1}(1-\sigma)\right]}{\Psi} x_{1}-x_{2}, \\
& \mathbf{J}_{21}=\frac{1}{\Pi}\left\{\alpha \eta(1-\phi)\left[\frac{\alpha(1+\gamma)(1-\phi)}{\Psi}-1\right] x_{1}+\frac{\alpha \theta_{2}(1+\gamma)(1-\sigma)[1-\eta(1-\phi)]}{(1-\eta) \Psi} \mathbf{J}_{11}\right\}, \\
& \mathbf{J}_{22}=\frac{1}{\Pi}\left\{\frac{\alpha \eta(\alpha-1)(1-\phi)^{2}\left[1-\theta_{1}(1-\sigma)\right]}{\Psi} x_{1}+\frac{\alpha \theta_{2}(1+\gamma)(1-\sigma)[1-\eta(1-\phi)]}{(1-\eta) \Psi} \mathbf{J}_{12}\right\},
\end{aligned}
$$

together with

$$
\Psi=\frac{\theta_{2}(1-\alpha)(\sigma-1)[1-\eta(1-\phi)]}{1-\eta}+1+\gamma-(1-\alpha)(1-\phi)
$$

and

\footnotetext{
${ }^{8}[1-\alpha(1-\phi)]>0$ is ensured by the lower bound of $\phi \geq 0$ together with $0<\alpha<1$.
} 


$$
\Pi=\frac{\left[1-\theta_{1}(1-\sigma)\right][1+\gamma-(1-\alpha)(1-\phi)]}{\Psi} .
$$

It follows that the determinant and trace of the model's Jacobian matrix $\mathbf{J}$ are

$$
\text { Det }=\frac{\alpha \eta(1-\phi) \Omega}{\left[1-\theta_{1}(1-\sigma)\right][(1-\alpha)(1-\phi)-(1+\gamma)]} x_{1} x_{2},
$$

where

$$
\Omega=\sigma(1-\alpha)(1-\phi)+\gamma[1-\alpha(1-\phi)]+\phi\left[1-\frac{\theta_{2}(1-\alpha)(1-\sigma)}{1-\eta}\right]
$$

and

$$
\operatorname{Tr}=\rho+\frac{\theta_{2}(1-\sigma)(1+\gamma)[1-\eta(1-\phi)]\{\rho+[1-\alpha(1-\phi)] \delta\}}{(1-\eta)(1-\phi)\left[1-\theta_{1}(1-\sigma)\right][(1-\alpha)(1-\phi)-(1+\gamma)]} .
$$

The economy's local stability property is determined by comparing the eigenvalues of $\mathbf{J}$ that have negative real parts with the number of initial conditions in the dynamical system (16), which is one because $c_{t}$ is a non-predetermined jump variable. As a result, the steady displays saddle-path stability and equilibrium uniqueness if and only if the two eigenvalues of $\mathbf{J}$ are of opposite signs $(\operatorname{Det}<0)$. If both eigenvalues have negative real parts ( Det $>0$ and $\operatorname{Tr}<0$ ), then the steady state is a locally indeterminate sink that can be exploited to generate endogenous cyclical fluctuations driven by agents' self-fulfilling expectations or sunspots. When both eigenvalues have positive real parts (Det $>0$ and $\operatorname{Tr}>0$ ), the steady state becomes a completely unstable source whereby any trajectory that diverges away from it may settle down to a limit cycle or to some more complicated attracting sets.

\subsection{When $0<\sigma<1$ and $0<\phi<1$}

In this case, $C_{t}$ and $G_{t}$ enter the household utility (4) as Edgeworth complements, and the tax schedule (6) is progressive. Since $0<\alpha, \phi<1$ and $\gamma \geq 0$, the term $[(1-\alpha)(1-\phi)-(1+\gamma)]$ in the denominator of (19) is negative. This finding, together with $0<\eta, \theta_{1}, \sigma<1$ and $x_{1}$, $x_{2}>0$, implies that the model's Jacobian matrix $\mathbf{J}$ possesses a positive determinant when $\Omega$ given by (20) is negative, i.e.

$$
\theta_{2}>\theta_{2}^{D e t} \equiv \frac{(1-\eta)\{\phi+\sigma(1-\alpha)(1-\phi)+\gamma[1-\alpha(1-\phi)]\}}{\phi(1-\alpha)(1-\sigma)},
$$

where $\theta_{2} \in(0,1)$ and $\theta_{2}^{\text {Det }}$ denotes the level of government-spending preference externality at which $\Omega=$ Det $=0$. 
Proposition. Under (i) utility complementarity between private and public consumption expenditures and (ii) progressive income taxation, the necessary and sufficient condition for our model economy to exhibit equilibrium indeterminacy and belief-driven business cycles is given by $(22)$.

Proof. See the Appendix that the inequality reported in (22) not only leads to a positive determinant, but also guarantees a negative trace, indicating the presence of two eigenvalues with negative real parts $($ Det $>0$ and $T r<0)$

The intuition for the above indeterminacy result can be understood as follows. Start the economy from its steady state, and consider a slight deviation caused by agents' optimistic anticipation about an expansion of future economic activities. Acting upon this belief, households will consume less and invest more today, which in turn lead to increases in future aggregate output (because of higher levels of capital and labor inputs in production), private consumption and income tax rate in that the fiscal policy rule is progressive. Through the government's balanced-budget constraint (12), the level of public spending also rises, which will then generate a further increase in future private consumption since $C_{t}$ and $G_{t}$ are Edgeworth complements in the household's utility function. For this alterative path to be justified as a self-fulfilling equilibrium, the after-tax return on investment $\left(1-\tau_{m t}\right) r_{t}$ must be monotonically increasing with respect to higher private consumption expenditures. Using equations (1), (6), (7), (8), (9) and (12), it can be shown that the aforementioned requisite condition is satisfied as long as $\Omega<0$ or Det $>$ o, i.e. the government-spending preference externality $\theta_{2}$ is sufficiently strong to satisfy the inequality as in (22). Consequently, agent's initial rosy expectations about the economy's future are validated in equilibrium. If the degree of preference externality from public consumption is not high enough to meet condition (22), and thus $\Omega>0$ or Det $<0$, our model's steady state will be a locally determinate saddle point.

In sharp contrast to previous studies with wasteful government purchases of goods and services (e.g. Schmitt-Grohé and Uribe, 1997; Guo and Lansing, 1998; and Christiano and Harrison, 1999), the arrow in Figure 1 illustrates that when $\frac{(1-\eta)(1+\gamma)}{(1-\alpha)(1-\sigma)}<\theta_{2}<1$, raising the tax progressivity $\phi$ ceteris paribus will eventually transform the steady state from a saddle point into a sink. It follows that unlike traditional Keynesian-type stabilization policies, a more progressive tax schedule may operate as an "automatic destabilizer" in our model economy by generating endogenous business cycle fluctuations, provided the level of public-spending preference externality is sufficiently high. As it turns out, this result also holds true in a onesector RBC model with productive government spending a la Chen and Guo (2013a); or in a one-sector representative-agent model of endogenous growth with productive flow of public 
expenditures a la Chen and Guo (2013b).

\subsection{When $\sigma>1$ and $0<\phi<1$}

In this case, $C_{t}$ and $G_{t}$ enter the household utility (4) as Edgeworth substitutes, and the tax schedule (6) is progressive. It is straightforward to show that the eigenvalues of the Jacobian matrix $\mathbf{J}$ display opposite signs $($ Det $<0)$, indicating the presence of saddle-path stability and equilibrium uniqueness. Therefore, when agents become optimistic and decide to raise their investment spending today, the mechanism described in the proceeding subsection that makes for multiple equilibria, i.e. an increase in the equilibrium after-tax marginal product of capital, will not be realized in that higher public expenditures now lower the marginal utility of private consumption. This implies that given the initial capital stock $k_{0}$, the period-0 level of the household's private consumption $c_{0}$ is uniquely determined to place the model economy on the convergent path toward its steady state $\left(k^{*}, c^{*}\right)$, and always stays there without any possibility of deviating transitional dynamics. As a result, equilibrium indeterminacy and belief-driven cyclical fluctuations can never occur in this setting, regardless of the strength of public-consumption preference externality.

\subsection{Special Cases}

Our analysis also allows for a rich set of theoretical possibilities regarding the macroeconomic (in)stability effects of progressive or flat income taxation within a one-sector representative agent model and helps bring together some recent findings in the RBC-based indeterminacy literature. First, we recover the result of Guo and Harrison (2008, section 3.2.2) under utility-generating government spending $\left(\theta_{2}>0\right)$ that is complementary to private consumption $(0<\sigma<1)$, indivisible labor $(\gamma=0)$ and a flat tax schedule $(\phi=0)$. It is straightforward to show that within this specification, the model's Jacobian matrix has a positive determinant when

$$
\left(\theta_{1}+\theta_{2}\right)(1-\sigma)>1
$$

which turns out to the necessary and sufficient condition for the Guo-Harrison economy to possess an indeterminate steady state. ${ }^{9}$

Next, it can be shown that our model exhibits saddle-path stability and equilibrium uniqueness either when there is no public-spending preference externality $\theta_{2}=0$, no matter whether the tax progressivity is positive or zero (as in the horizontal axis of Figure 1); or when the fiscal

\footnotetext{
${ }^{9}$ Since $0<\sigma<1$ under utility complementarity between private and public consumptions, satisfying condition (23) requires that $\theta_{1}+\theta_{2}>1$, which is not considered in the current paper.
} 
policy rule is flat $\phi=0$, regardless of private and public consumption expenditures being Edgeworth complements (as in the vertical axis of Figure 1) or substitutes in the household utility. In both cases, the after-tax marginal product of capital will not rise in response to agents' belief-driven investment spurts, thus preventing their optimistic expectations from becoming self-fulfilling. It follows that as in a prototypical one-sector RBC model under laissez-faire, the economy does not display endogenous business cycles caused by changes in agents' animal spirits.

Finally, when $\theta_{2}=\phi=0$, our model collapses to one with useless government purchases and a constant income tax rate, as in Guo and Harrison (2004). In this formulation, it is straightforward to show that its Jacobian's determinant is negative, thus the eigenvalues of the log-linearized dynamical system (16) are of opposite signs and local determinacy always prevails.

\section{Conclusion}

This paper has explored the theoretical interrelations between a progressive tax schedule and equilibrium (in)determinacy in an otherwise standard one-sector real business cycle model with balanced budget and utility-generating government purchases of goods and services. Under utility complementarity between private and public consumption expenditures together with progressive income taxation, we analytically show that the economy possesses an indeterminate steady state if and only if the degree of government-spending preference externality is higher than a critical value. In contrast to a conventional automatic stabilizer, raising tax progressivity may destabilize this formulation of our model by generating endogenous belief-driven cyclical fluctuations. We also find that the economy always exhibits saddle-path stability and equilibrium uniqueness under utility substitutability between private and public consumptions together with progressive income taxation. Finally, the same stability/uniqueness result continues to hold when there is no preference externality from government purchases, regardless of the level of tax progressivity under consideration; or when the fiscal policy rule is flat, no matter whether private and public consumption expenditures are Edgeworth complements or substitutes in the household's utility function.

This paper can be extended in several directions. For example, it would be worthwhile to examine our model economy with national debt (i.e. non-balanced budget) a la SchmittGrohé and Uribe (1997, p. 990), or multiple production sector a la Guo and Harrison (2001), or sustained endogenous growth a la Fernández, Novales and Ruiz (2004). In addition, we can 
incorporate features that are commonly considered in the New-Keynesian literature, such as nominal price/wage rigidities and investment adjustment costs, among others. These possible extensions will allow us to study the robustness of this paper's theoretical results and policy implications, as well as further enhance our understanding of the dynamic (in)stability effects of progressive income taxation in representative-agent models with utility-generating government spending. We plan to pursue these research projects in the future. 


\section{Appendix}

Proof of Proposition. Figure 1 plots the combinations of $\phi$ (the tax progressivity) and $\theta_{2}$ (the degree of preference externality from public consumption) that graphically characterize our model's local stability properties under $0<\sigma, \phi<1$. Using equations (22) and (19), it is straightforward to show that (i) $\frac{\partial\left(\theta_{2}^{D e t}\right)}{\partial \phi}<0$ and $\frac{\partial^{2}\left(\theta_{2}^{D e t}\right)}{\partial \phi^{2}}>0$, hence the locus of Det $=0$ is negatively sloped and convex to the origin; and (ii) $\frac{\partial(D e t)}{\partial \theta_{2}}>0$ thus the area above (below) the downward-sloping curve $\operatorname{Det}=0$ exhibits a positive (negative) determinant. ${ }^{10}$

Next, we find that the level of government-spending preference externality, denoted as $\theta_{2}^{T r} \in(0,1)$, at which the Jacobian's trace (21) equals zero is given by

$$
\theta_{2}^{T r}=\frac{\sigma \rho(1-\eta)(1-\phi)[1+\gamma-(1-\alpha)(1-\phi)]}{\mu_{1}(1-\sigma)}
$$

and that

$$
\frac{\partial\left(\theta_{2}^{T r}\right)}{\partial \phi}=\frac{\theta_{2}^{T r}(1+\gamma)\left(\delta \mu_{2}+\rho \mu_{3}\right)}{\mu_{1}(1-\phi)[1+\gamma-(1-\alpha)(1-\phi)]} \gtrless 0
$$

where

$$
\begin{gathered}
\mu_{1} \equiv \delta(1+\gamma)[1-\alpha(1-\phi)][1-\eta(1-\phi)]+\rho\left[\phi(1+\gamma)+(1-\alpha)(1-\eta)(1-\phi)^{2}\right]>0, \\
\mu_{2} \equiv(1-\alpha)(1-\phi)[2-(\alpha+\eta)(1-\phi)]-(1+\gamma)\left[1-\alpha \eta(1-\phi)^{2}\right] \gtrless 0,
\end{gathered}
$$

and

$$
\mu_{3} \equiv(1-\alpha)(1-\phi)[2-\eta(1-\phi)]-(1+\gamma) \gtrless 0 .
$$

\footnotetext{
${ }^{10}$ Given the parametric restriction of $\phi, \theta_{2} \in(0,1)$, both the vertical and horizontal intercepts for the locus of $D e t=0$ need to lie between zero and one as well. When $\phi=1$, we find that $(1-\eta)<(1-\alpha)$, i.e. the steady-state government spending to output ratio is lower than the labor share of national income, is required
} to satisfy $0<\frac{(1-\eta)(1+\gamma)}{(1-\alpha)(1-\sigma)}<1$. Using $\alpha<\eta$ and $\theta_{2}=1$, it can be shown that for the $\phi$-intercept,

$$
\frac{(1-\eta)(1-\alpha)(\gamma+\sigma)}{\eta(1-\alpha)(1-\sigma)-\alpha(1-\eta)(1+\gamma)}>0
$$

since its denominator $\eta(1-\alpha)(1-\sigma)-\alpha(1-\eta)(1+\gamma)$ is positive; and that

$$
\frac{(1-\eta)(1-\alpha)(\gamma+\sigma)}{\eta(1-\alpha)(1-\sigma)-\alpha(1-\eta)(1+\gamma)}-1=\frac{(1-\eta)(1+\gamma)-(1-\alpha)(1-\sigma)}{\eta(1-\alpha)(1-\sigma)-\alpha(1-\eta)(1+\gamma)}<0
$$


It follows that the exact shape and curvature for the locus of $\operatorname{Tr}=0$ cannot be analytically determined. However, using (21), it is straightforward to show that $\frac{\partial(T r)}{\partial \theta_{2}}<0$, hence points above (below) the nonlinear curve $T r=0$ possess a negative (positive) trace.

Finally, we prove that $\theta_{2}^{D e t}>\theta_{2}^{T r}$ under this parameterization with $0<\sigma, \phi<1^{11}$. As a result, the locus of $T r=0$ (regardless of its shape and curvature) will lie entirely below the downward-sloping and convex curve Det $=0$ depicted in Figure 1. This implies that the region of Det $>0$ is completely subsumed by that with $\operatorname{Tr}<0$. Therefore, condition (22) not only leads to a positive determinant, but also guarantees a negative trace, thus the steady state is a (locally indeterminate) sink.

${ }^{11}$ In particular, the difference between $\theta_{2}^{D e t}$ and $\theta_{2}^{T r}$ is given by

$$
\theta_{2}^{D e t}-\theta_{2}^{T r}=\frac{(1-\eta)\left(\rho \Delta_{1}+\delta \Delta_{2}\right)}{\Delta_{3}},
$$

where

$\Delta_{1} \equiv \sigma(1-\alpha)^{2}(1-\phi)^{2}[1-\eta(1-\phi)]+\left[\phi(1+\gamma)+(1-\alpha)(1-\eta)(1-\phi)^{2}\right]\{\phi+\gamma[1-\alpha(1-\phi)]\}>0$,

$\Delta_{2} \equiv(1+\gamma)[1-\alpha(1-\phi)][1-\eta(1-\phi)]\{\phi+\gamma[1-\alpha(1-\phi)]+\sigma(1-\alpha)(1-\phi)\}>0$, and

$\Delta_{3} \equiv \phi(1-\alpha)(1-\sigma)\left\{\delta(1+\gamma)[1-\eta(1-\phi)][1-\alpha(1-\phi)]+\rho\left[\phi(1+\gamma)+(1-\alpha)(1-\eta)(1-\phi)^{2}\right]\right\}>0$.

Since $\eta, \delta \in(0,1)$ and $\rho>0, \theta_{2}^{D e t}$ is always higher than $\theta_{2}^{T r}$. 


\section{References}

[1] Agénor, P-R. (2011), "Schooling and Public Capital in a Model of Endogenous Growth," Economica 78, 108-132.

[2] Baier, S.L. and G. Glomm (2001), "Long-Run Growth and Welfare Effects of Public Policies with Distortionary Taxation," Journal of Economic Dynamics and Control 25, 2007-2042.

[3] Barro, R.J. (1990), "Government Spending in a Simple Model of Endogenous Growth," Journal of Political Economy 98, S103-S125.

[4] Barro, R.J. and X. Sala-i-Martin (1992), "Public Finances in Models of Economic Growth," Review of Economic Studies 59, 645-661.

[5] Bean, C. R. (1986), "The Estimation of Surprise Models and the Surprise Consumption Function," Review of Economic Studies 53, 497-516.

[6] Benhabib, J. and R.E.A. Farmer (1999), "Indeterminacy and Sunspots in Macroeconomics," in J. Taylor and M. Woodford, eds., Handbook of Macroeconomics, Amsterdam: North Holland, 387-448.

[7] Campbell, J.Y. and G.N. Mankiw (1990), "Permanent Income, Current Income, and Consumption," Journal of Business and Economic Statistics 8, 265-279.

[8] Cazzavillan, G. (1996), "Public Spending, Endogenous Growth, and Endogenous Fluctuations," Journal of Economic Theory 71, 394-415.

[9] Chen, B-L. (2006), "Public Capital, Endogenous Growth, and Endogenous Fluctuations," Journal of Macroeconomics 28, 768-774.

[10] Chen, S-H. and J-T. Guo (2013a), "Progressive Taxation and Macroeconomic (In)stability with Productive Government Spending," Journal of Economic Dynamics and Control 37, 951-963.

[11] Chen, S-H. and J-T. Guo (2013b), "On Indeterminacy and Growth under Progressive Taxation and Productive Government Spending," forthcoming in Canadian Journal of Economics.

[12] Christiano, L.J. and S.G. Harrison (1999), "Chaos, Sunspots and Automatic Stabilizers," Journal of Monetary Economics 44, 3-31.

[13] Fernández, E., A. Novales and J. Ruiz (2004), "Indeterminacy under Non-Separability of Public Consumption and Leisure in the Utility Function," Economic Modelling 21, 409-428.

[14] Futagami, K., Y. Morita and A. Shibata (1993), "Dynamic Analysis of an Endogenous Growth Model with Public Capital," Scandinavian Journal of Economics 95, 607-625.

[15] Greiner, A. (2006), "Progressive Taxation, Public Capital, and Endogenous Growth," FinazArchiv 62, 353-366.

[16] Greiner, A. (2007), "An Endogenous Growth Model with Public Capital and Sustainable Government Debt," Japanese Economic Review 58, 345-361.

[17] Glomm G. and B. Ravikumar (1994), "Public Investment in Infrastructure in a Simple Growth Model," Journal of Economic Dynamics and Control 18, 1173-1187.

[18] Glomm G. and B. Ravikumar (1997), "Productive Government Expenditures and LongRun Growth," Journal of Economic Dynamics and Control 21, 183-204. 
[19] Guo, J-T. and S.G. Harrison (2001), "Tax Policy and Stability in a Model with SectorSpecific Externalities," Review of Economic Dynamics 4, 75-89.

[20] Guo, J-T. and S.G. Harrison (2004), "Balanced-Budget Rules and Macroeconomic (In)stability," Journal of Economic Theory 119, 357-363.

[21] Guo, J-T. and S.G. Harrison (2008), "Useful Government Spending and Macroeconomic (In)stability under Balanced-Budget Rules," Journal of Public Economic Theory 10, 383397.

[22] Guo, J-T. and K.J. Lansing (1998), "Indeterminacy and Stabilization Policy," Journal of Economic Theory 82, 481-490.

[23] Hu, Y., R. Ohdoi and K. Shimomura (2008), "Indeterminacy in a Two-Sector Endogenous Growth Model with Productive Government Spending," Journal of Macroeconomics 30, 1104-1123.

[24] Hansen, G.D. (1985), "Indivisible Labor and the Business Cycle," Journal of Monetary Economics 16, 309-327.

[25] Ni, S. (1995), "An Empirical Analysis on the Substitutability between Private Consumption and Government Purchases," Journal of Monetary Economics 36, 593-605.

[26] Palivos, T., C.Y. Yip and J. Zhang (2003), "Transitional Dynamics and Indeterminacy of Equilibria in an Endogenous Growth Model with a Public Input," Review of Development Economics 7, 86-98.

[27] Rogerson, R. (1988), "Indivisible Labor, Lotteries, and Equilibrium," Journal of Monetary Economics 21, 3-16.

[28] Schmitt-Grohé, S. and M. Uribe (1997), "Balanced-Budget Rules, Distortionary Taxes and Aggregate Instability," Journal of Political Economy 105, 976-1000.

[29] Slobodyan, S. (2006), "One Sector Models, Indeterminacy and Productive Public Spending," CERGE-EI Working Paper Series No. 293.

[30] Turnovsky, S.J. (1997), "Fiscal Policy in a Growing Economy with Public Capital," Macroeconomic Dynamics 1, 615-639.

[31] Turnovsky, S.J. (1999), "Productive Government Expenditures in a Stochastically Growing Economy," Macroeconomic Dynamics 3, 544-570.

[32] Zhang, J. (2000), "Public Services, Increasing Returns, and Equilibrium Dynamics," Journal of Economic Dynamics and Control 24, 227-246. 


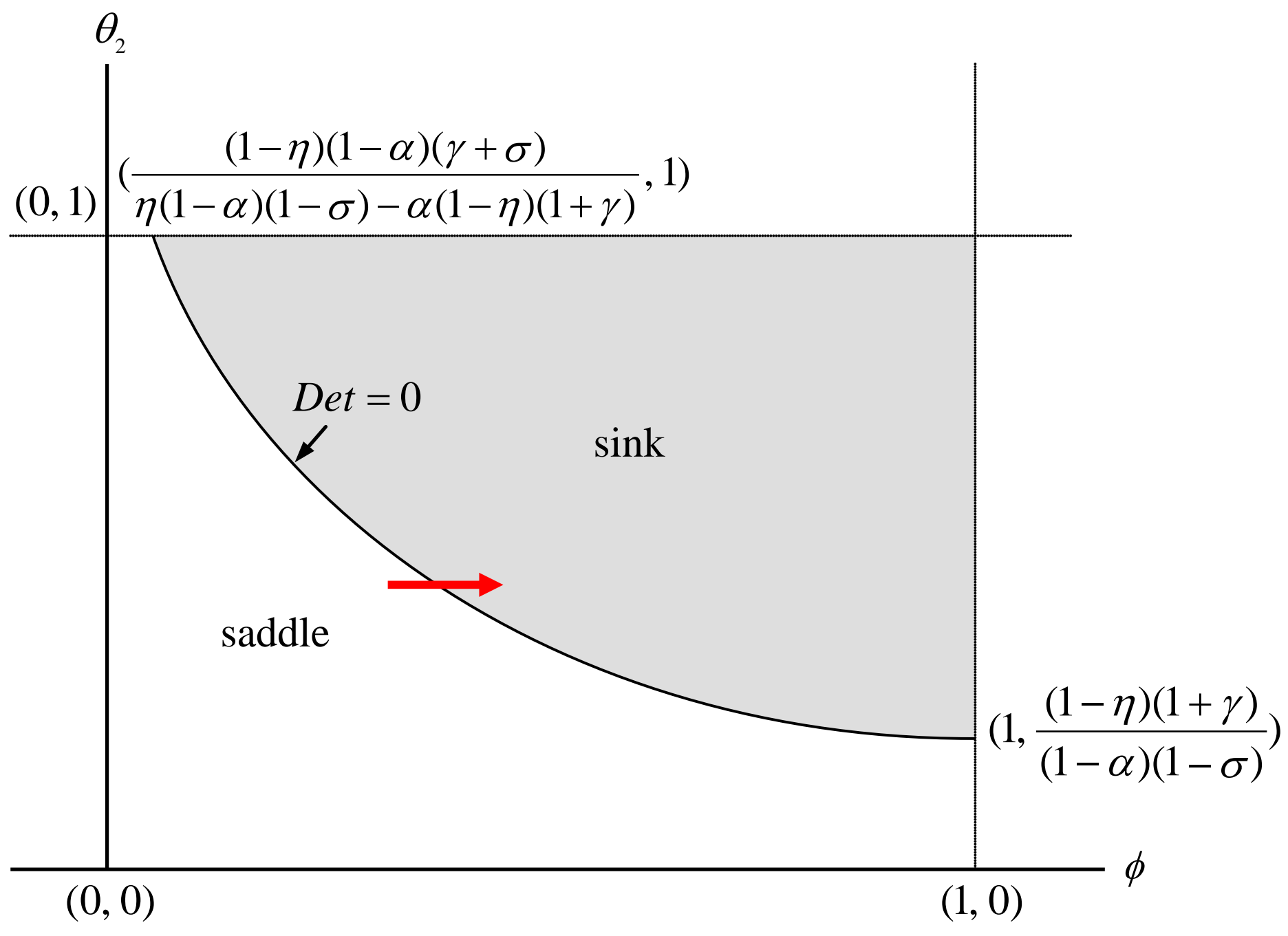

Figure 1: $0<\sigma<1$ and $0<\phi<1$ 